\section{Regards sur l'économie allemande}

Bulletin économique du CIRAC

$90 \mid 2009$

Varia

\title{
L'innovation, priorité absolue pour les PME industrielles
}

Isabelle Bourgeois

\section{OpenEdition}

Édition électronique

URL : http://journals.openedition.org/rea/3567

DOI : $10.4000 /$ rea.3567

ISBN : 978-2-8218-0876-8

ISSN : 1965-0787

Éditeur

CIRAC

Édition imprimée

Date de publication : 1 mars 2009

Pagination : 7-14

ISSN : 1156-8992

Référence électronique

Isabelle Bourgeois, «L'innovation, priorité absolue pour les PME industrielles », Regards sur l'économie allemande [En ligne], 90 I mars 2009, mis en ligne le 01 mars 2011, consulté le 19 avril 2019. URL

http://journals.openedition.org/rea/3567; DOI : 10.4000/rea.3567 


\title{
L'innovation, priorité absolue pour les PME industrielles
}

\author{
Isabelle Bourgeois
}

Si elle affecte le moral des entreprises industrielles allemandes qui voient s'effondrer leurs commandes, la crise ne les amène pas à baisser les bras en matière d'innovation. Bien au contraire, comme le révèle l'enquête DIHK-Innovationsreport 2008/2009 de la Fédération des chambres de commerce et d'industrie allemandes. Près de la moitié des PME innovantes interrogées prévoient en effet d'accroître leurs efforts en 2009, comptant mettre à profit la période de crise actuelle pour développer leur compétitivité et consolider, voire accroître à terme leurs parts de marché mondiales. Si elles envisagent d'investir dans la $R \& D$ et de recruter, elles voient pourtant leur potentiel bridé par un certain nombre de handicaps structurels auxquels les pouvoirs publics devraient remédier d'urgence, résumait Martin Wansleben, président de la fédération DIHK, lors de la présentation de l'enquête à la fin 2008. Trois axes s'imposent à l'action politique, rappelle-t-il. Les deux premiers sont spécifiques aux PME : revoir le régime fiscal et réduire les charges bureaucratiques (voir REA 89/08). Le troisième concerne toutes les entreprises, quelle que soit leur taille : remédier au manque de chercheurs et d'ingénieurs-techniciens hautement qualifiés. Mais les PME, elles aussi, doivent fournir des efforts : mieux valoriser leur potentiel en se dotant notamment de méthodes professionnelles de management de l'innovation leur permettant d'accéder plus facilement au crédit bancaire.

Or cette enquête de la DIHK, dont les conclusions s'appuient implicitement sur les travaux menés par diverses autres institutions, montre surtout que les PME industrielles allemandes partagent une approche de l'innovation qui dépasse de loin le seul concept de R\&D. Mais comme tout understatement, cette acception ne s'éclaire qu'à la lumière des nombreux travaux consacrés outre-Rhin au concept d'innovation. Et ce n'est qu'en replaçant l'enquête DIHK dans son contexte qu'on s'aperçoit à quel point l'étonnante confiance des PME allemandes en cette période de crise est entretenue par des facteurs culturels.

\section{Une dynamique qui appelle une politique d'innovation mieux structurée pour le Mittelstand}

L'innovation reste plus que jamais une priorité pour les PME industrielles innovantes : $46,9 \%$ d'entre elles comptent étendre leurs efforts en la matière et à peu près autant $(48,4 \%)$ les maintenir à niveau constant. L'enquête de la DIHK avait été réalisée au paroxysme de la crise financière et au moment où les entreprises commençaient à voir s'assombrir leurs perspectives. Certes, la détérioration du contexte global les rend plus prudentes pour 2009, comme le montre l'écart avec leurs réponses concernant les efforts réalisés en matière d'innovation au cours des douze mois précédant l'enquête : la majorité des sociétés (54\%) affirme les avoir développés, et la plupart des autres (42,9\%) les avoir maintenus à niveau constant. Néanmoins, dans l'ensemble, la dynamique d'innovation des PME industrielles allemandes reste intacte.

\begin{tabular}{|c|c|c|c|c|}
\hline \multicolumn{5}{|c|}{ En matière d'innovation, que projetez-vous pour les 12 mois à venir ? } \\
\hline Activités... & en hausse... & constantes... & en baisse... & Entr. non concernée \\
\hline Dépenses de R\&D & $42,2 \%$ & $47,3 \%$ & $5,6 \%$ & $4,9 \%$ \\
\hline Effectifs en R\&D & $32,5 \%$ & $54,6 \%$ & $4,1 \%$ & $8,8 \%$ \\
\hline Dépôt de brevets & $18,3 \%$ & $43,7 \%$ & $7,4 \%$ & $30,6 \%$ \\
\hline Recours à un fonds d'aide à la R\&D & $19,1 \%$ & $30,0 \%$ & $9,2 \%$ & $41,7 \%$ \\
\hline
\end{tabular}

Source des données : DIHK-Innovationsreport 2008/2009.

$95 \%$ des PME industrielles veulent continuer à innover en 2009 


\section{DIHK-Innovationsreport 2008/2009}

Ce sondage effectué par le réseau des $80 \mathrm{CCl}$ allemandes auprès des $\mathrm{PME}$ industrielles innovantes succède à une enquête menée en 2006 auprès des conseillers innovation des $\mathrm{CCl}$ qui avaient dressé un état des lieux de l'activité en termes de R\&D et d'innovation menée par les PME affiliées répertoriées comme innovantes, ainsi que les start-ups technologiques (DIHK-Innovationsreport 2007). Elle avait permis d'identifier le manque de main-d'œuvre qualifiée comme un frein majeur à la réalisation de l'objectif de $3 \%$ du PIB consacré à la R\&D fixé dans le cadre de la Stratégie de Lisbonne. Elle avait, de même, révélé un important "problème d'image " affectant les programmes nationaux et européens de soutien à la R\&D : les PME les ignorent largement; et ceux qu'elles connaissent ne répondent pas à leurs besoins et/ou impliquent une telle charge administrative qu'elles préfèrent ne pas y recourir (voir REA 83/07).

L'enquête DIHK-Innovationsreport 2008-2009 repose, elle, sur des sondages effectués par les CCI entre la mi-octobre et la fin novembre 2008 auprès d'un échantillon représentatif de 530 entreprises innovantes. Parmi celles-ci figuraient 370 PME de moins de 250 salariés et 160 de plus de 250 salariés. Les sociétés interrogées se recrutent dans les quatre branches phares de l'industrie : construction mécanique, automobile, chimie et électrotechnique, qui totalisent traditionnellement $80 \%$ des dépenses privées de R\&D en Allemagne (DIHK). Dans l'industrie, le taux d'entreprises innovantes, c'est-à-dire dont les stratégies d'innovation produit ou process sont couronnées de succès, est de $58 \%$ (données 2007, source : ZEW, 2009).

Les plus actives sont les plus petites...

... et celles qui bénéficient d'un programme de soutien à l'innovation
Toutefois, les intentions varient selon la taille des entreprises. Ce sont les plus grandes (plus de 250 salariés) qui se montrent les plus prudentes; elles commencent en effet, dès l'automne 2008, à rencontrer des difficultés pour financer leurs projets d'investissements lourds et risqués. Alors qu'elles sont $58 \%$ à avoir accru leur effort de R\&D l'an passé et $5 \%$ à l'avoir diminué (soit 53 points d'écart), le solde des réponses positives se réduit à 41 points en ce qui concerne l'activité à venir. Les plus petites (moins de 250 salariés), touchées elles aussi par la chute des commandes, mais souvent moins directement, maintiennent mieux leur cap d'innovation, le solde positif de leurs réponses pour 2009 ne baissant que de 8 points pour s'établir à 42 points. Assurément, les liens personnels de confiance qu'elles entretiennent avec leur conseiller de la Sparkasse ou de la banque coopérative locale (voir $R E A 88 / 08$ ) facilitent leur accès au crédit. Mais bien plus décisive encore semble leur nette préférence pour l'autofinancement de la $R \& D$, qui garantit l'autonomie des choix stratégiques (voir REA 83/07).

L'enquête révèle également que ce sont les entreprises qui bénéficient d'un des nombreux programmes publics d'aide à l'innovation (leur part est de $58 \%$ sur l'ensemble de la RFA, et même de $85 \%$ dans les nouveaux Länder) qui se montrent les plus actives. $42 \%$ d'entre elles envisagent de recruter pour développer leur R\&D (contre $18 \%$ seulement de celles qui n'ont accès à aucun programme). La DIHK en conclut que, du moins en ce qui concerne les entreprises qui recourent aux mesures publiques, les politiques allemandes de soutien à l'innovation remplissent visiblement leurs objectifs : dès lors qu'elles sont soutenues, les entreprises étendent leurs activités de R\&D et accroissent en conséquence leurs effectifs.

\begin{tabular}{|c|c|c|c|c|c|c|}
\hline Effets ... & très positifs... & positifs... & nuls... & négatifs... & très négatifs... & Mesures non connues \\
\hline Politiques des Länder & $4,7 \%$ & $23,2 \%$ & $61,5 \%$ & $1,3 \%$ & $0,8 \%$ & $8,5 \%$ \\
\hline Politiques du Bund & $5,1 \%$ & $25,5 \%$ & $59,2 \%$ & $1,9 \%$ & $0,2 \%$ & $8,2 \%$ \\
\hline Politiques européennes & $1,9 \%$ & $13,9 \%$ & $70,9 \%$ & $2,1 \%$ & $0,8 \%$ & $10,5 \%$ \\
\hline
\end{tabular}

Source des données : DIHK-Innovationsreport 2008/2009.

Ces programmes étant largement inadaptés aux besoins des PME, ...

... le gouvernement fédéral en a lancé un nouveau en 2008 : ZIM
II n'en reste pas moins que l'accès à ces programmes est considéré comme difficile par les PME, surtout celles de moins de 250 salariés (78\% des aides publiques vont aux grandes entreprises ; EFI, 2008). D'abord, leur grande diversité s'assimile à leurs yeux à une véritable « jungle inextricable ». Et lorsqu'elles ont réussi à identifier un programme à leur mesure, elles sont rebutées par la complexité de sa mise en œuvre et une paperasse qui implique une charge de travail incompatible avec les impératifs de la production au quotidien. La logique administrative des financements est elle aussi inadaptée : pour lancer le projet de R\&D, il faut attendre que l'accord soit notifié, ce qui se traduit par des délais interdisant toute réactivité au marché. S'y s'ajoute une relation défavorable entre le montant des aides (faibles) et le coût administratif lié à leur obtention (beaucoup trop élevé en proportion).

L'enquête réalisée par la fédération DIHK sur ce sujet en 2007 (DIHK-Innovationsreport 2007), et qui avait fait apparaître le peu d'efficacité de la politique de soutien de l'UE, du Bund et des Länder à l'innovation dans les PME (sauf pour le programme PRO INNO // visant le renforcement de la coopération avec les acteurs de 
la recherche), a incité les pouvoirs politiques à commencer à réviser celle-ci. C'est ainsi que le gouvernement fédéral avait lancé le $1^{\text {er }}$ juillet 2008 un nouveau programme : Zentrales Innovationsprogramm Mittelstand (ZIM), courant jusqu'à la fin 2013. Dans le cadre du second programme de soutien à la conjoncture décidé le 13 janvier 2009, le budget dédié au programme ZIM a été plus que doublé pour 2009/2010, passant à 1,5 milliard $€$. Ajouté aux autres programmes de soutien à l'innovation dans le Mittelstand du ministère fédéral de l'Economie, le total des aides aux PME innovantes sera de 2,2 milliards $€$ durant ces deux années.

Le programme Zentrales Innovationsprogramm Mittelstand - ZIM

Visant à développer la proximité de la $R \& D$ avec le marché, ce programme fédéral de soutien à l'innovation dans les PME conjugue la politique générale de soutien au Mittelstand et la politique de soutien à l'innovation technologique. S'inspirant de mesures qui avaient fait leurs preuves dans les nouveaux Länder, il s'applique à l'ensemble du territoire allemand, à toutes les branches et à toutes les technologies. II a pour objectif de promouvoir des projets de R\&D menés en partenariat et impliquant la constitution de réseaux, y compris transnationaux ; il intègre donc les clusters. Depuis 2009 s'y ajoute le soutien à des projets développés isolément par une PME (ouest de la RFA) et même (à l'ouest comme à l'est) par une grosse société occupant jusqu'à 1000 salariés, la Commission européenne ayant donné son accord. L'objectif de ces dispositions nouvelles, limitées aux années 2009 et 2010, est de permettre de soutenir 7000 programmes de R\&D supplémentaires. Le programme ZIM (www.zim-bmwi.de) se compose de trois modules :

(1) projets de coopération entre plusieurs PME, entre des PME et des centres de recherche appliquée. Dans la limite d'un plafond de $350000 €$ par projet, le soutien public s'échelonne entre $35 \%$ et $50 \%$ du coût prévisionnel du projet, le taux maximal étant réservé aux PME de moins de 50 salariés. Les centres de recherche impliqués se voient, eux, verser jusqu'à $100 \%$ du coût. Gestionnaire : Arbeitsgemeinschaft industrieller Forschungsvereinigungen 'Otto von Guericke' e.V. AiF ;

(2) projets de constitution de réseaux de six PME au moins. Le soutien est alors destiné aux prestations liées au management et à l'organisation du réseau. Dans la limite d'un plafond de $350000 €$, les charges salariales du manager de réseau sont subventionnées à hauteur de $70 \%$ la première année, de $50 \%$ la deuxième et de $30 \%$ la troisième. Gestionnaire : VDI/VDE Innovation und Technik GmbH ;

(3) projet isolé présenté par une PME et visant le développement de produits, process et prestations techniques innovants. La contribution ZIM couvre dans ce cas entre $25 \%$ et $45 \%$ du coût total (maximum : $350000 €$ ), les PME est-allemandes continuant de percevoir les subventions les plus élevées. Gestionnaire : EuroNorm GmbH.

S'y ajoute, pour les modules (1) et (3), un soutien aux activités de conseil et prestations liées au développement du projet (études de marché, veille en matière de brevets, etc.) à proportion de $50 \%$ au maximum des frais engagés (plafond : $50000 €$ par projet). Les projets sont sélectionnés par un jury composé de 15 experts issus des milieux scientifiques et économiques.

L'approche du programme ZIM est construite sur un des facteurs structurels qui nourrissent le dynamisme d'innovation des acteurs privés en Allemagne : la culture de la coopération et du partenariat en réseaux, conjuguant utilement celle-ci avec l'approche que promeut la politique d'innovation (et de clusters) européenne déclinée à l'échelle nationale (voir dans ce numéro l'exemple du «Cluster Sensorik »). Le programme ZIM est également un exemple classique de la logique d'offre qui caractérise la politique allemande de soutien à l'innovation et où les pouvoirs publics se contentent de mettre à disposition des acteurs privés des moyens leur permettant de réaliser plus facilement les projets définis par eux-mêmes (logique de bottom up). A eux ensuite de mettre à profit les outils professionnels de management de l'innovation que propose le marché - surtout pour le financement des projets comme la gestion des brevets, les deux points faibles des PME.

\begin{tabular}{|c|c|c|c|c|}
\hline \multicolumn{5}{|c|}{ Votre entreprise procède-t-elle systématiquement au management de l'innovation dans les domaines suivants? } \\
\hline Toutes entreprises & oui & parfois & non & prévu \\
\hline Développement de projets / créativité & $38,6 \%$ & $44,8 \%$ & $13,3 \%$ & $3,3 \%$ \\
\hline Evaluation et planification & $37,0 \%$ & $42,3 \%$ & $17,1 \%$ & $3,6 \%$ \\
\hline Financement & $31,8 \%$ & $31,0 \%$ & $32,5 \%$ & $4,7 \%$ \\
\hline Marketing/vente & $44,2 \%$ & $32,6 \%$ & $20,0 \%$ & $3,2 \%$ \\
\hline Management de la coopération dans les processus liés à l'innovation & $24,5 \%$ & $36,1 \%$ & $35,2 \%$ & $4,2 \%$ \\
\hline Gestion des brevets & $32,1 \%$ & $22,9 \%$ & $41,5 \%$ & $3,4 \%$ \\
\hline Culture interne de l'innovation & $31,2 \%$ & $43,9 \%$ & $20,3 \%$ & $4,6 \%$ \\
\hline Entreprises de moins de 250 salariés & oui & parfois & non & prévu \\
\hline Développement de projets / créativité & $36,2 \%$ & $44,9 \%$ & $15,0 \%$ & $3,9 \%$ \\
\hline Evaluation et planification & $33,1 \%$ & $42,5 \%$ & $21,2 \%$ & $3,2 \%$ \\
\hline Financement & $27,5 \%$ & $32,5 \%$ & $34,7 \%$ & $5,3 \%$ \\
\hline Marketing/vente & $42,9 \%$ & $33,2 \%$ & $20,1 \%$ & $3,8 \%$ \\
\hline Management de la coopération dans les processus liés à l'innovation & $24,0 \%$ & $31,3 \%$ & $39,2 \%$ & $5,4 \%$ \\
\hline Gestion des brevets & $22,1 \%$ & $23,2 \%$ & $50,7 \%$ & $4,0 \%$ \\
\hline Culture interne de l'innovation & $27,6 \%$ & $42,6 \%$ & $24,9 \%$ & $4,9 \%$ \\
\hline
\end{tabular}


Selon l'enquête 2008/2009 de la fédération DIHK, le programme ZIM semble dans l'ensemble adapté aux besoins des PME. Le recalibrage intervenu au début 2009 (extension du soutien aux projets isolés) permettra d'apporter en outre un correctif au problème du financement initial de projets innovants que rencontrent encore nombre de PME, start-ups technologiques ou entreprises en développement. Ce problème avait été accru par la réforme de la fiscalité des entreprises (2008) qui limite notamment la possibilité de reporter sur l'exercice suivant les pertes induites par l'investissement dans une jeune pousse high-tech. Ces dispositions, conjuguées à d'autres modifications fiscales (voir REA 89/08), risquent d'aggraver les effets de la crise et doivent être revues d'urgence.

\section{Principal frein à l'innovation industrielle : la pénurie d'ingénieurs et techniciens}

Globalement, les pouvoirs publics du Bund et des Länder semblent prendre la mesure des besoins particuliers des PME et commencent à réviser leurs programmes pour en accroître l'efficience, du moins en ce concerne les incitations financières. Un problème de fond demeure néanmoins : « les mesures de soutien ne peuvent produire leurs effets qu'à la condition qu'il y ait un volet de main-d'œuvre qualifiée à recruter en R\&D » (DIHK 2008/2009). Or c'est là que le bât blesse le plus cruellement. Les entreprises se plaignent de plus en plus massivement de la pénurie d'ingénieurs et de techniciens. Comment donc accroître les effectifs de R\&D (actuellement de 315000 en équivalent temps plein) si, selon le DIHK-Innovationsreport 2007 par exemple, il en manque au moins 30000 à l'horizon 2010 - et au moins 6000 dans les seules petites entreprises ? II n'y a alors rien d'étonnant à ce que près de $57 \%$ des entreprises interrogées à la fin 2008 soulignent que le principal handicap à l'innovation qu'elles rencontrent est qu'elles ne trouvent pas la maind'œuvre aux compétences requises.

\begin{tabular}{|lcc}
\hline \multicolumn{2}{|c|}{ Aux yeux de votre entreprise, où se situent les principaux handicaps à l'innovation au sein du site Allemagne ? } \\
Entreprises & Toutes & $<250$ salariés \\
Disponibilité de la main-d'œuvre qualifiée & $56,4 \%$ & $54,1 \%$ \\
Accès aux fonds de soutien publics & $31,8 \%$ & $32,5 \%$ \\
Possibilités de coopérer avec des centres de recherche & $9,8 \%$ & $11,0 \%$ \\
Bureaucratie & $54,5 \%$ & $57,2 \%$ \\
Accès au financement & $26,7 \%$ & $31,0 \%$ \\
Réforme 2008 de la fiscalité des entreprises & $15,7 \%$ & $19,9 \%$ \\
Autres & $6,7 \%$ & $6,5 \%$ \\
\hline
\end{tabular}

Source des données : DIHK-Innovationsreport 2008/2009. *) plusieurs réponses possibles.

Une enquête de 2007 révèle une forte pénurie d'ingénieurs et techniciens dans l'industrie

Les PME manquent de sortants du système de formation continue...
Ces réponses confirment le constat d'une autre enquête de la fédération DIHK, réalisée en 2007 auprès de 20000 entreprises, essentiellement des PME (dont $35 \%$ dans l'industrie) et consacrée au manque général de main-d'œuvre qualifiée (Kluge Köpfe - vergeblich gesucht!). Tous secteurs confondus et quelle que soit la taille de l'entreprise, le manque le plus criant concerne les ingénieurs et techniciens : $54 \%$ des entreprises offrant des postes vacants cherchent vainement à en recruter. Dans l'industrie, où $40 \%$ des entreprises se plaignent d'un manque général de main-d'œuvre qualifiée, ce taux atteint même $83 \%$ en ce qui concerne les ingénieurs et techniciens. Les branches les plus affectées y sont la métallurgie (manque général : $46 \%$, manque d'ingénieurs-techniciens : $96 \%$ ), la construction mécanique (respectivement $61 \%$ et $94 \%$ ) et la construction automobile ( $46 \%$ et $90 \%$ ). Manifestement, relève l'étude, " malgré d'excellentes perspectives d'emploi, les métiers scientifiques et techniques ont un problème d'image ».

Si la pénurie concerne tous les niveaux de qualification, on observe néanmoins deux tendances. D'une part, la croissance d'une demande non satisfaite de sortants du système de formation professionnelle, qu'elle soit initiale ou, plus encore, continue. Dans l'industrie, près d'une entreprise sur deux cherche ainsi désespérément des titulaires d'un brevet de maîtrise (Meister) ou d'un diplôme équivalent sanctionnant une formation continue ; en 2001, une sur cinq seulement. Or depuis, la proportion des actifs qui en dispose n'a pas varié ; elle est toujours de 
l'ordre de $10 \%$. La forte hausse de la demande montre à quel point ces qualifications sont prisées outre-Rhin. Si elles sont aussi recherchées que les diplômes de l'enseignement supérieur (du bachelor au master), elles ont sur ces derniers un avantage compétitif : les compétences acquises sont non seulement directement opérationnelles, mais elles apportent aussi la garantie d'une aptitude foncière du salarié à évoluer - donc à innover -, une compétence clef pour la compétitivité, surtout pour les petites structures dont la culture interne de réactivité au marché est assise sur les compétences non interchangeables de ses collaborateurs. Comme, traditionnellement, les PME industrielles moyennes (moins de 200 salariés) sont fortement impliquées dans la formation duale, initiale et continue, la pénurie est moindre, les entreprises ayant développé des efforts considérables pour flexibiliser les voies d'accès aux différents métiers et pour rendre plus efficaces les passerelles vers la formation continue.

\begin{tabular}{|c|c|c|c|c|c|}
\hline \multicolumn{6}{|c|}{ Difficultés de recrutement rencontrées par les entreprises selon les niveaux de qualification recherchés } \\
\hline Profil recherché & $\begin{array}{c}\text { Sans } \\
\text { qualification }\end{array}$ & $\begin{array}{l}\text { Sortants du } \\
\text { système dual }\end{array}$ & $\begin{array}{l}\text { Brevet de maîtrise } \\
\text { ou équival. *) }\end{array}$ & $\begin{array}{c}\text { Bachelor ou } \\
\text { Fachhochschule }\end{array}$ & $\begin{array}{l}\text { Master ou } \\
\text { équival. }\end{array}$ \\
\hline \multicolumn{6}{|c|}{ Selon les secteurs } \\
\hline Industrie & $8 \%$ & $38 \%$ & $40 \%$ & $45 \%$ & $29 \%$ \\
\hline BTP & $12 \%$ & $45 \%$ & $41 \%$ & $24 \%$ & $15 \%$ \\
\hline Commerce & $12 \%$ & $57 \%$ & $35 \%$ & $16 \%$ & $10 \%$ \\
\hline Services & $20 \%$ & $43 \%$ & $28 \%$ & $35 \%$ & $31 \%$ \\
\hline Tous secteurs & $13 \%$ & $43 \%$ & $35 \%$ & $36 \%$ & $26 \%$ \\
\hline \multicolumn{6}{|c|}{ Selon la taille de l'entreprise (nombre de salariés) } \\
\hline $1-9$ & $16 \%$ & $47 \%$ & $29 \%$ & $26 \%$ & $19 \%$ \\
\hline $10-19$ & $18 \%$ & $47 \%$ & $31 \%$ & $26 \%$ & $16 \%$ \\
\hline $20-199$ & $13 \%$ & $45 \%$ & $36 \%$ & $34 \%$ & $23 \%$ \\
\hline $200-999$ & $7 \%$ & $34 \%$ & $40 \%$ & $54 \%$ & $42 \%$ \\
\hline$>1000$ & $6 \%$ & $24 \%$ & $33 \%$ & $60 \%$ & $57 \%$ \\
\hline
\end{tabular}

Source des données : DIHK, Kluge Köpfe - vergeblich gesucht !, automne 2007. *) exclusivement formation continue.

La seconde tendance : parmi les diplômés du supérieur, c'est dans les filières MINT (mathématiques, sciences de l'ingénieur) qu'il y a la plus forte pénurie. Dans l'industrie, elle se concentre sur les diplômés de niveau bachelor ou les sortants d'un établissement d'enseignement supérieur spécialisé proposant des formations courtes (Fachhochschule). Cette pénurie frappe plus particulièrement les sociétés de plus de 1000 salariés. Dans l'ingénierie informatique et l'électrotechnique, le taux des postes difficiles à pourvoir dans cette catégorie atteint respectivement $64 \%$ et $62 \%$. Quant aux diplômés de l'université de niveau master ou équivalent, ils font défaut à un quart des entreprises rencontrant des problèmes de recrutement, la plus forte pénurie s'observant dans l'industrie pharmaceutique (73\%), les TIC (63\%), l'ingénierie informatique (53\%), les technologies médicales ou les mesures et asservissement (52\%), ainsi que la chimie (46\%).

C'est là le résultat d'une forte baisse des inscriptions dans les filières MINT depuis une dizaine d'années (15\% seulement des étudiants allemands aujourd'hui ; voir REA 88/08). Entre 1995 et 2004, par exemple, le nombre de diplômés de ces filières a reculé de $40 \%$ dans les secteurs de l'électrotechnique, de la chimie ou de la construction mécanique (DIHK-Innovationsreport 2007). Or le problème n'est pas seulement quantitatif. II est encore plus qualitatif : les entreprises regrettent que la formation universitaire soit " trop peu ouverte sur l'innovation, la culture du risque et le sens de l'entreprise » (DIHK, 2008/2009).

La DIHK plaide dès lors pour sensibiliser les jeunes aux "phénomènes naturels » dès le primaire, rendre obligatoire une épreuve de sciences de la nature au baccalauréat et mieux orienter les étudiants dans ces filières. Mais elle ne se contente pas de formuler des préconisations. A l'automne 2008, les $\mathrm{CCl}$ allemandes ont lancé un portail mettant en réseau enseignants du secondaire, PME innovantes et acteurs de la formation professionnelle afin de développer dans l'enseignement et la formation les contenus scientifiques et techniques (www.ihk-tecnopedia.de) et, ce faisant, contribuer à susciter les vocations.

Les entreprises étant largement responsables de la formation professionnelle (les PME industrielles forment $80 \%$ des apprentis ; voir REA 75/06), elles ont multiplié
... les grandes sociétés de diplômés du supérieur

Recul des inscriptions dans les filières MINT et formation inadéquate

Les $\mathrm{CCl}$ cherchent dès lors à susciter les vocations...

... les PME s'intéressent aux seniors et aux femmes... 
... l'Etat vise une hausse globale des qualifications leurs efforts en matière de formation initiale et, surtout, continue, commencé à exploiter le potentiel que représente l'emploi des seniors (surtout les PME de moins de 20 salariés où, plus qu'ailleurs, l'expérience individuelle est une compétence clef) comme l'emploi féminin ou à recruter des salariés étrangers.

A l'adresse des pouvoirs publics, la fédération DIHK plaide pour des mesures facilitant l'accueil de salariés étrangers et, bien sûr, pour une politique résolue de modernisation du système de formation. Lors du «Sommet de l'éducation et de la formation » qui s'était tenu le 22 octobre 2008 à Dresde, Bund et Länder avaient adopté une « Initiative de qualification » (Qualifizierungsinitiative für Deutschland) venant en complément du Programme national de réformes 2008-2010 soumis à la Commission européenne dans le cadre de la Stratégie de Lisbonne. Diverses mesures y étaient adoptées pour notamment améliorer la qualité du système universitaire, assurer une meilleure fluidité entre formation professionnelle et supérieure, développer la formation continue ou accroître le nombre d'étudiants dans les filières scientifiques et techniques. Cette politique qui vise une hausse globale des qualifications doit se traduire par une part de $10 \%$ du PIB consacré à la formation et à la recherche en 2015. Dans le cadre du second programme conjoncturel (Konjunkturpaket II) décidé le 13 janvier 2009 par le gouvernement fédéral, le Bund met à disposition 6,5 milliards € supplémentaires afin de cofinancer (à hauteur de $65 \%$ ) les investissements des Länder et des communes pour moderniser les structures du système éducatif - de la maternelle à l'université.

Le manque de main-d'œuvre qualifiée n'est certes pas nouveau, et les milieux politiques comme économiques ont commencé à prendre conscience au cours des dernières années de l'importance stratégique d'une main-d'œuvre qualifiée pour une économie qui ne dispose pas d'autre ressource naturelle, et où le vieillissement démographique contribue à la raréfaction de cette ressource clef. Mais la résolution du problème prend une urgence nouvelle dans la crise actuelle où la mobilisation de tous les acteurs est plus que jamais requise pour préserver la compétitivité des entreprises et, de ce fait, la compétitivité industrielle du site Allemagne, tributaire des biens et services de hautes et moyennes technologies. L'élan innovateur des $\mathrm{PME}$ allemandes en tout cas est intact, comme le révèle le DIHK-Innovationsreport 2008/2009. Ce qu'elles attendent actuellement des pouvoirs publics, ce ne sont pas tant des aides financières. Elles leur demandent de faire en sorte que le cadre des activités soit favorable à l'innovation : en réduisant la paperasse, en supprimant les dispositions désincitatives pour l'investissement contenues dans le nouveau régime de fiscalité des entreprises (voir REA 89/08), en calant mieux les politiques de soutien à l'innovation sur les besoins des entreprises et, priorité absolue, en luttant contre le manque de main-d'œuvre qualifiée et de chercheurs.

\section{En Allemagne, ce sont les PME qui tirent l'innovation}

"Seuls les Hommes sont innovants. La technologie n'est pas innovante, elle est tout au plus le fruit de l'innovation ». C'est en ces termes que Heinz Putzhammer, membre du bureau exécutif du DGB, plaidait, en 2004 déjà, pour une politique générale de hausse des qualifications à l'aube de l'économie du savoir, contribuant ainsi à fonder l'axe principal d'une politique syndicale rénovée. Et il plaidait pour une participation active des partenaires sociaux à la consolidation de la compétitivité du site Allemagne dans une économie globalisée.

“En quoi l'innovation concerne-t-elle les syndicats ? " s'intitulait la plaquette du DGB diffusant le plaidoyer de H. Putzhammer. De fait, ils sont impliqués dans le système de formation professionnelle comme dans la formation des représentants des salariés élus aux Conseils d'établissement présents dans toutes les entreprises de plus de 5 salariés; ces conseils ayant un large pouvoir consultatif dans les questions relatives à l'organisation de la production ou de l'établissement (voir $R E A$ 72/05), ils sont directement concernés par toutes les formes d'innovation comme le développement de nouveaux modèles économiques (par exemple la conception d'une offre intégrée produits/services) ou de nouveaux modèles d'organisation interne. Ensuite, les syndicats sont cofondateurs du RKW Rationalisierungs- und Innovationszentrum der Deutschen Wirtschaft, une association qui a pour mission d'accompagner les PME dans leur stratégie de compétitivité, en matière de R\&D comme d'organisation (voir REA 74/05). A ce rôle institutionnel s'ajoute un constat : dans plus des deux tiers des PME, les compétences en matière d'innovation sont liées à une personne et donc indissociables de l'individu qui les porte (Fraunhofer ISI, 2005). Cette dépendance, qui est le propre des petites structures, risque de pénaliser les PME dans leur développement durable. 
L'acception large du concept d'innovation qu'il défendait sous-tend aujourd'hui la plupart des études publiées sur le sujet outre-Rhin (et, plus récemment, dans l'UE), à commencer par celles des Instituts Fraunhofer, plus particulièrement chargés d'accompagner les PME. Et elle fonde l'approche holistique de la politique allemande d'innovation depuis le second gouvernement Schröder (voir REA 74/05).

\begin{abstract}
La Commission d'experts Recherche et Innovation (EFI), think tank indépendant institué en 2006 par le gouvernement fédéral pour évaluer les structures et le potentiel allemand en la matière et formuler des préconisations à l'intention des pouvoirs publics, prend elle aussi ce concept dans son sens large : «il désigne les nouveautés techniques, organisationnelles, sociales ou autres dont la mise en œuvre est tentée ou couronnée de succès". Il s'agit d'un "ensemble global de processus dynamiques, souvent résumé sous le terme de 'destruction créatrice' ” (rapport 2008). Et les experts rappellent que, dans cette approche, " la R\&D n'est qu'une composante parmi d'autres des processus d'innovation. L'innovation inclut en effet également toutes les démarches liées à la commercialisation ou la valorisation, et qui ne sont pas comptabilisées dans la R\&D ». II est vrai que celles-ci sont difficilement quantifiables. Les efforts d'innovation des entreprises hors de la R\&D stricto sensu sont dès lors largement négligés, explique de son côté le tableau de bord de l'innovation dans l'UE qui vient de paraître en janvier 2009 (EIS 2008) et qui leur consacre un chapitre ("Neglected innovators »), rappelant : "R\&D is not the only method of innovating ".

L'intensité de la R\&D, c'est-à-dire, dans le cas d'un Etat, la part des dépenses publiques et privées rapportées au PIB (la part des dépenses par rapport au chiffre d'affaires dans le cas d'une entreprise), utilisée comme unité de mesure de la compétitivité des économies dans les comparaisons statistiques européennes et internationales, ne reflète donc qu'un aspect de l'innovation. En Allemagne, selon le rapport 2008 de la DG Recherche de la Commission européenne, l'intensité de la R\&D était de $2,53 \%$ en 2006 (de 2,09 \% en France et de 1,84\% dans l'UE 27) ; le secteur privé allemand assurait 67,6 \% des dépenses (52,2\% en France et $54,6 \%$ dans I'UE 27), ce qui correspond à une intensité de $1,77 \%$ du PIB selon les données de l'EFI.

En 2007, les dépenses privées de R\&D ont crû de 2,8\% pour atteindre 53,5 milliards $€$; selon toutes prévisions, elles devraient avoir augmenté de $6,2 \%$ en 2008 , passant à 56,8 milliards $€$. A la mi-2008, les entreprises allemandes prévoyaient de porter ces dépenses à 59,3 milliards € en 2009 (Stifterverband für die deutsche Wissenschaft, décembre 2008).
\end{abstract}

Un constat empirique corrobore, si besoin en était, cette approche large : ce sont les PME qui tirent l'innovation industrielle outre-Rhin. Une lecture sous le seul angle de la R\&D ne permet pas de discerner cet état de fait : ce sont en effet les grosses entités qui réalisent l'essentiel de l'effort de $R \& D$, rappelle le rapport 2008 de l'EFI. Actuellement, les sociétés moyennes (de 100 à 500 salariés) ne sont que $29 \%$ à pratiquer la R\&D, ce taux tombant à $12 \%$ pour les plus petites (moins de 100 salariés). Mais les PME (de 5 à 500 salariés) montent en puissance, comme le révèle l'étude sur la R\&D en Allemagne (FuE-Datenreport 2007) menée en 2005 auprès de 30000 entreprises par le Stifterverband für die deutsche Wissenschaft, une fédération de mécènes dont l'une des activités est notamment d'établir statistiques et analyses pour le gouvernement fédéral, l'UE ou I'OCDE. Alors qu'en 2003, seulement $11,1 \%$ des dépenses privées de R\&D leur étaient imputables, cette part s'élevait à $12,9 \%$ en 2005 ; dans le même temps, la part des dépenses effectuées par les sociétés de plus de 10000 salariés tombait de $51,4 \%$ à $47,5 \%$. Et, ainsi que le souligne pour sa part le rapport 2008 de I'EFI, depuis 1998, la proportion des PME industrielles innovantes disposant de leur propre division de R\&D est passée de quelque $50 \%$ à plus des deux tiers.

$\mathrm{Si}$ on considère, comme le fait l'EFI, non plus la seule R\&D mais l'effort d'innovation (où les activités de R\&D entrent environ pour moitié), alors les PME allemandes réalisent $28 \%$ des dépenses en la matière. C'est peu, certes (d'où l'abondance des politiques de soutien à l'innovation dans le Mittelstand), mais cela classe tout de même l'Allemagne dans le haut des palmarès internationaux. Alors que le taux des entreprises innovantes s'établit à $90 \%$ parmi celles qui occupent plus de 500 salariés, il tombe à $70 \%$ en dessous. II atteint tout de même encore $51 \%$ parmi les petites PME (de 5 à 49 salariés). Le fait que le taux moyen d'entreprises innovantes soit de $58 \%$ dans l'industrie allemande s'explique donc principalement par l'omniprésence des PME dans le tissu économique (quelque $99 \%$ des sociétés), ce qui amène l'EFI à souligner dans son rapport 2008 que les PME « sont déterminantes pour la diffusion large et l'ancrage en profondeur de l'innovation, de la recherche et du développement dans l'économie " allemande. Leur rôle est d'autant plus fondamental pour l'avenir de la compétitivité du «site Allemagne » qu'elles sont également créatrices de lien social: elles forment, elles embauchent et, ce faisant, elles dispensent une identité sociale.

VISIBLEMENT, LE MITTELSTAND ALLEMAND GARDE CONFIANCE dans ses atouts fonciers que sont sa réactivité à l'évolution de la demande, son souci d'offrir des produits et
La faible part des PME dans les dépenses de R\&D masque...

... leur rôle déterminant pour la diffusion et l'ancrage de l'innovation 
prestations de qualité, sa culture du partage des savoirs au sein de réseaux de partenariat et, par-dessus tout, sa culture construite sur une vision à long terme et sur le respect de la compétence humaine (voir $R E A$ 84/07). Encore faut-il que la relève soit assurée pour que perdurent ces facteurs à l'origine de la capacité à innover qui caractérise les PME industrielles allemandes et qui expliquent pourquoi l'Allemagne est aussi bien placée en comparaison européenne. Le risque que fait peser sur leur avenir le manque de main-d'œuvre qualifiée relativise dès lors celui d'un resserrement du crédit ou la perspective d'une baisse de l'activité induite par la chute des entrées de commandes. Leur confiance est aussi le fruit de l'expérience : le Mittelstand industriel a surmonté les crises de 1993 puis de 2003 en se restructurant, en rationalisant sa production et son organisation - en un mot : en innovant au sens plein du terme, ce qui lui avait permis non seulement de ne pas perdre le contact avec son marché, mais plus encore de gagner en compétitivité.

C'est peut-être là que se trouve le cœur du message de confiance implicite que contient l'enquête 2008/2009 de la DIHK : le Mittelstand industriel innovant continue de miser sur sa propre compétitivité, saisissant la crise comme une chance lui permettant de mieux se positionner pour l'avenir. Certes, les entreprises interrogées ne réaliseront certainement pas toutes les intentions formulées à l'automne 2008, faute de commandes ou de moyens. Mais l'essentiel n'est pas là. Car ce qu'elles révèlent par leurs réponses, c'est avant tout une attitude profondément ancrée dans la culture économique allemande et qu'on ne peut comprendre réellement que si on replace ce sondage dans son contexte plus général : les risques actuels ne leur font en rien perdre de vue leurs objectifs sur le long terme. La confiance et l'attitude proactive des PME industrielles allemandes sont d'origine systémique.

\section{Indications bibliographiques}

- ARmbruster H. et al., « Innovationskompetenz auf wenigen Schultern. Wie abhängig sind Betriebe vom Wissen und den Fähigkeiten einzelner Mitarbeiter? », Fraunhofer ISI, Mitteilungen aus der Produktionsinnovationserhebung, $\mathrm{n}^{\circ} 35$, février 2005

- Bourgeols I., «Comment l'intelligence vient aux PME allemande », Regards sur l'économie allemande, $n^{\circ} 74$, décembre 2005

- DIHK, Deutsche Unternehmen setzen auf Innovationen - trotz Finanzmarktkrise. DIHK-Innovationsreport 2008/2009, décembre 2008, Kluge Köpfe - vergeblich gesucht! Fachkräftemangel in der deutschen Wirtschaft, novembre 2007, Fachkräftebedarf und Image von FuE-Förderprogrammen auf dem Prüfstand, DIHK-Innovationsreport 2007, août 2007 (www.dihk.de)

- European Commission, EuRopean Research AREA, A more research-intensive and integrated European Research Area. Science, Technology and Competitiveness key figures report 2008/2009 (http://ec.europa.eu/research/research-eu)

- European Innovation Scoreboard (EIS) 2008. Comparative Analysis of Innovation performance, rapport realisé par le Centre de recherche UNU-MERIT pour le compte d'INNO METRICS, janvier 2009 (http://www.proinno-europe.eu/metrics)

- EXPERTENKOMMISSION FORSCHUNG UND INNOVATION (EFI), Gutachten zu Forschung, Innovation und technologischer Leistungsfähigkeit 2008, février 2008 (www.e-fi.de)

- HazouARD S., « Innovation: l'industrie allemande leader en Europe », Regards sur l'économie allemande, $\mathrm{n}^{\circ} 81$, mai 2007

- « Industrieforschung und Konjunktur im Gleichschritt. FuE-Quote auch 2007 unverändert », facts Forschung \& Entwicklung, décembre 2008 (www.stifterverband.de)

- «Innovation: Mehr als Forschung und Entwicklung », Fraunhofer ISI, Mitteilungen aus der Produktionsinnovationserhebung, $\mathrm{n}^{\circ} 33$, mai 2004

- Innovationsverhalten der deutschen Wirtschaft. Indikatorenbericht zur Innovationserhebung 2008, ZEW, infas, Fraunhofer ISI, janvier 2009 (www.zew.de)

- KINKEL S. et al., « Innovation : Mehr als Forschung und Entwicklung. Wachstumschancen auf anderen Innovationspfaden », Fraunhofer ISI, Mitteilungen aus der Produktionsinnovationserhebung, $\mathrm{n}^{\circ} 33$, mai 2004

- Putzhammer H., «Plädoyer für einen erweiterten Innovationsbegriff », in « Innovation - was geht das die Gewerkschaften an ? ", Materialien und Argumente n¹, juin 2004, www. dgb.de - StifTerVerband für die Deutsche Wissenschaft, FuE-Datenreport 2007. Tabellen und Daten, Essen, 2007 (www.stifterverband.org)

- Uppenberg K., R\&D in Europe. Expenditures across Sectors, Regions and Firm Sizes, Centre for European Policy Studies, Bruxelles, 2009 (www.ceps.eu)

- ZEW, Innovationsverhalten der Unternehmen in Deutschland 2006. Aktuelle Entwicklungen und ein internationaler Vergleich, Studien zum deutschen Innovationssystem, n4/2008, janvier 2008 (www.zew.de). 